\title{
Le genre : une « catégorie utile » de l'histoire juive allemande (1967-1933)
}

Geschlecht: eine "nützliche Kategorie“ deutsch-jüdischer Geschichte (1867-1933)

Gender: a "Useful Category" of German-Jewish History (1867-1933)

\section{Patrick Farges}

\section{(2) OpenEdition}

\section{Journals}

Édition électronique

URL : https://journals.openedition.org/ceg/7614

DOI : $10.4000 /$ ceg. 7614

ISSN : 2605-8359

\section{Éditeur}

Presses Universitaires de Provence

\section{Édition imprimée}

Date de publication : 25 septembre 2019

Pagination : 171-183

ISBN : 979-10-320-0234-6

ISSN : 0751-4239

Référence électronique

Patrick Farges, «Le genre : une « catégorie utile » de l'histoire juive allemande (1967-1933) », Cahiers d'Études Germaniques [En ligne], 77 | 2019, mis en ligne le 25 mars 2021, consulté le 15 juin 2021. URL : http://journals.openedition.org/ceg/7614 ; DOI : https://doi.org/10.4000/ceg.7614 


\title{
Le genre : une « catégorie utile » de l'histoire juive allemande (1867-1933)
}

\author{
Patrick FARGES \\ Université de Paris / ICT (EA 337)
}

La dimension genrée de I'histoire juive allemande fait l'objet d'études depuis plus de trente ans ${ }^{1}$. Si l'outil analytique "genre », défini comme interaction sociale entre les sexes, a été proposé au début des années 1960 aux États-Unis, il s'agit d'un concept voyageur entre les disciplines et les aires culturelles ${ }^{2}$. Dans les années 1970, il a d'abord migré vers les sciences sociales puis, dans les années 1980, vers I'histoire : ainsi, l'article fondateur de Joan Scott, auquel nous empruntons notre titre, est publié dans l'American Historical Review en $1986^{3}$. Sa réception s'est opérée rapidement tant en France - la traduction française date de 1988 - que dans l'espace germanophone, dans sa version originale, puis en traduction $\left(1994^{4}\right)$. Il faut néanmoins attendre les années 1990 (pour l'Allemagne) et 2000 (pour la France ${ }^{5}$ ) pour que le genre soit le cœur d'analyses historiques. Si l'article de Scott est souvent cité en référence, il n'est pas toujours lu. L'historienne y affirme l'« utilité » du genre comme « catégorie d'analyse historique » et énonce ses références théoriques, parfois très éloignées des instruments de l'histoire du genre telle qu'elle est pratiquée aujourd'hui : d'une part les écrits de Michel Foucault sur I'histoire de la sexualité; d'autre part la psychanalyse, en ce qu'elle

1. Voir Jonathan Frankel (dir.), Jews and Gender: the Challenge to Hierarchy, Oxford/ New York, Oxford UP, 2000; Marion Kaplan, Deborah Dash Moore (dir.), Gender and Jewish History, Bloomington, Indiana UP, 2011 ; Leora Auslander, Sylvie Steinberg (dir.), Dossier « Judaïsme(s) : genre et religion », Clio. Femmes, Genre, Histoire 44, 2016.

2. Il en a ipso facto été modifié. Voir Anne Isabelle François, Patrick Farges, Perin Emel Yavuz, «Les Gender Studies entre transfert et institutionnalisation : une circulation des modèles et des pouvoirs », in Aline Le Berre, Angelika Schober, Florent Gabaude (dir.), Le pouvoir au féminin. Identités, représentations et stéréotypes dans l'espace germanique, Limoges, PU Limoges, 2013, p. 27-42.

3. Joan W. Scott, « Gender: A Useful Category of Historical Analysis », American Historical Review 91, 1986, p. $1053-1075$.

4. «Genre : une catégorie utile d'analyse historique » (trad. Éleni Varikas), Les Cahiers du GRIF 3738, 1988, p. 125-153; "Gender: eine nützliche Kategorie der historischen Analyse » (trad. Robin Mitchell), in Nancy Kaiser (dir.), Selbst bewusst. Frauen in den USA, Leipzig, Reclam, 1994, p. 27-75.

5. Toutefois, comme le fait remarquer Anne-Marie Sohn à propos de la réception française de l'article de Scott, les « spécialistes de l'histoire des femmes [étaient] sensibles aux implications du concept de gender bien avant la francisation du terme ». Anne-Marie Sohn, "Écrire l'Histoire des femmes et du genre en France », Historiens \& Géographes 392, 2005, p. 78. 
permet de comprendre la formation du sujet ${ }^{6}$. Selon Scott, c'est à travers le langage comme pratique sociale que se construit l'identité genrée. Or avec le temps et l'usage, le genre est passé d'une histoire des pratiques de langage et de nomination (autant de dimensions encore présentes dans l'article de Scott) à une histoire des pratiques et relations sociales, donc d'une forme d'histoire culturelle à une histoire sociale. Dans le même temps, l'objet de l'histoire « du genre » s'est déplacé : d'une histoire des femmes à une histoire des relations de sexe et de genre. Françoise Thébaud énumère les différentes étapes de cette évolution :

une histoire identitaire des femmes portée par le mouvement de libération des femmes, une histoire de l'oppression ou de la révolte féminine, [...] une histoire de la différence sexuelle ou des relations entre les hommes et les femmes, une histoire attentive à cerner la construction sociale du masculin et du féminin, une histoire des différences et des processus de différenciation soucieuse d'articuler des identités multiples ${ }^{7}$.

La question du genre en histoire juive s'est posée au sujet des liens entre judaïsme et féminisme, mais aussi dans des études relevant de l'histoire sociale des femmes et des familles juives en Europe centrale et orientale ${ }^{8}$. Notons la place qu'y prend le " cas » allemand (et autrichien), hypertrophié, qui éclipse souvent les expériences juives dans d'autres aires : dans le « croisement ${ }^{9}$ » entre histoire juive et histoire du monde germanophone, le pôle " allemand » est souvent connoté comme étant la norme, le pôle " juif » étant marqué du sceau de la différence. Or les enjeux de genre permettent de mieux saisir les frictions qui se produisent à l'occasion du croisement ${ }^{10}$ et nous souhaitons montrer qu'ils permettent d'éclairer d'un jour nouveau certaines questions de I'histoire des Juifs et Juives en Allemagne entre 1867 et $1933^{11}$, notamment celle de l'assimilation (et de son opposé, la dissimilation ${ }^{12}$ ) ou encore celle du processus d'entrée en modernité;

6. Joan W. Scott, Gender and the Politics of History, New York, Columbia UP, 1988.

7. Françoise Thébaud, Écrire I'histoire des femmes et du genre, Lyon, ENS éditions, 2007, p. 174.

8. David Kraemer (dir.), The Jewish Family: Metaphor and Memory, New York, Oxford UP, 1989 ; Nancy L. Green, "La femme juive. Formation et transformations », in Georges Duby, Michelle Perrot (dir.), Histoire des femmes en Occident, t. IV, Paris, Plon, 1991, p. 215-229; Marion Kaplan, The Making of the Jewish Middle Class: Women, Family, and Identity in Imperial Germany, New York, Oxford UP, 1991 (trad. Ingrid Strobl : Jüdisches Bürgertum. Frau, Familie und Identität im Kaiserreich, Hamburg, Dölling \& Galitz, 1997); Monika Richarz, « Frauen in Familie und Öffentlichkeit », in Steven M. Lowenstein, Paul Mendes-Flohr, Peter Pulzer, Monika Richarz (dir.), Deutsch-jüdische Geschichte in der Neuzeit, vol. 3, München, Beck, 1997, p. 69-100; Miriam Gebhardt, Das Familiengedächtnis. Erinnerung im deutsch-jüdischen Bürgertum 1890 bis 1932, Stuttgart, Franz Steiner, 1999.

9. Michael Werner \& Bénédicte Zimmermann (dir.), De la comparaison à I'histoire croisée, Paris, Seuil, 2004.

10. Sur I'histoire du genre dans l'espace germanophone, voir Ute Frevert, Women in German History: From Bourgeois Emancipation to Sexual Liberation, Oxford, Berg, 1989; Karen Hagemann, Frauenalltag und Männerpolitik: Alltagsleben und gesellschaftliches Handeln von Arbeiterfrauen in der Weimarer Republik, Bonn, Dietz, 1990.

11. Voir Kirsten Heinsohn, Stefanie Schüler-Springorum (dir.) Deutsch-jüdische Geschichte als Geschlechtergeschichte. Studien zum 19. und 20. Jahrhundert, Göttingen, Wallstein, 2006.

12. C'est Franz Rosenzweig qui, en 1922, transfère le terme linguistique de dissimilation à l'histoire du peuple juif. Comme il le note dans son journal en 1922, la dissimilation est un « désengagement par rapport à la civilisation occidentale » et un « retour aux sources de l'identité juive ». Voir 
le genre met également l'accent sur la capacité d'agir (agency ${ }^{13}$ ) des acteur-es qui, dans leur négociation quotidienne d'une appartenance allemande, restèrent distinctement fidèles à leur identité juive.

\section{Femmes et judaïsme}

La question des liens entre genre et judaïsme a été abordée dès les années 1970-80 par l'histoire des femmes, qui a mis en avant de nouvelles dimensions de l'histoire juive, comme la sphère domestique ou personnelle, notamment dans le champ de l'expression religieuse et de la spiritualité. La tradition juive assigne des places, des droits et devoirs différents aux femmes et aux hommes. Rappelons la prière matinale des hommes : "Béni sois-tu Seigneur Dieu de ne pas m'avoir fait femme car les femmes ne sont pas tenues d'accomplir les commandements ». Seuls les hommes ont l'accès au Livre et les femmes ont trois obligations rituelles : allumer les bougies, séparer un bout de pâte lors du shabbat et s'abstenir sexuellement après les menstruations. Jusque tardivement au XVIII siècle, la grande majorité de la population juive dans l'espace germanophone a vécu conformément aux préceptes religieux traditionnels, respectant une séparation des sexes ${ }^{14}$ marquée spatialement, notamment dans les lieux de culte.

Les choses sont toutefois plus complexes. D'une part, les femmes juives se rendaient à la synagogue à un rythme plus soutenu que ce que la loi rabbinique ne l'exigeait, faisant ainsi preuve d'une agentivité propre. D'autre part, la pratique religieuse des hommes comme performance sociale se déroulait devant un public féminin, qui validait et commentait pour la communauté les actes performés: dans des rôles différents, hommes et femmes concouraient à soutenir l'ordre social ${ }^{15}$. Dans son étude sur le rôle des femmes dans les familles juives bourgeoises, I'historienne Marion Kaplan met l'accent sur la culture domestique : depuis une position privée, les femmes exerçaient une influence sur la sphère publique. Le travail social et les responsabilités communautaires qu'elles assumaient leur fournissaient autant d'espaces d'action ${ }^{16}$. Comparant femmes juives et non juives, I'historienne Ute Frevert souligne que les femmes juives se consacraient plus largement aux affaires publiques que les non juives appartenant au même

Myriam Bienenstock, « Assimilation - Dissimilation. Rosenzweig sur l'école », in id. (dir.), Héritages de Franz Rosenzweig. « Nous et les Autres », Paris, Éd. de l'Éclat, 2011, p. 140-148.

13. L'agency (capacité d'agir ou agentivité) est la faculté des acteur-es à agir sur le monde et à le transformer. L'agency s'oppose à ce qu'imposent les structures. Voir Monique Haicault, « Autour d'agency. Un nouveau paradigme pour les recherches de genre », Rives méditerranéennes 41, 2012, p. 11-24.

14. Stefanie Schüler-Springorum, Geschlecht und Differenz. Perspektiven deutsch-jüdischer Geschichte, Paderborn, Schöningh, 2014, p. 16-17.

15. Voir Andreas Gotzmann, Jüdische Autonomie in der Frühen Neuzeit: Recht und Gemeinschaft im deutschen Judentum, Göttingen, Wallstein, 2008, p. 713-745.

16. Kaplan, The Making of the Jewish Middle Class, p. 16-17, 229. 
groupe social ${ }^{17}$. Par ailleurs, il faut souligner que l'attention portée à la position des femmes dans le judaïsme est un topos récurrent de l'antijudaïsme chrétien : évoquer le statut inférieur des femmes permettait aussi de mieux dénigrer le judaïsme ${ }^{18}$.

L'histoire des mondes juifs depuis la Haskala, née dans un contexte ashkénaze, est marquée par un débat continu entre tradition et modernité, entraînant des reconfigurations importantes dans l'« arrangement des sexes ${ }^{19}$ ». Dans l'espace germanophone du milieu du XIXe siècle, les défenseurs du judaïsme réformé néoorthodoxe insistent sur les valeurs familiales juives (Familieninnigkeit), révélant l'agir féminin et imposant de nouvelles injonctions aux hommes. La cellule familiale et la féminité sont alors placées au cœur des préoccupations identitaires de la minorité juive : forces de cohésion familiale et culturelle, les femmes jouent un rôle central dans la transmission du judaïsme ${ }^{20}$. Dans le même temps, on passe d'une religion des hommes à des formes de religiosité féminines : après avoir régressé dans les synagogues, l'observance juive se perpétue en privé. Mais cette idéologie a un effet pervers : on impute aux femmes l'affaiblissement du judaïsme.

Portées par les aspirations à la modernité, certaines femmes juives en Allemagne s'engagent à partir du dernier tiers du XIXe siècle dans les mouvements d'émancipation des femmes ${ }^{21}$. C'est dans la rencontre entre le mouvement féministe et le mouvement religieux libéral, tous deux ouverts à des formes de modernité, que sont nées les revendications des femmes pratiquantes demandant l'accès aux textes sacrés, aux études et à des lieux de pratique religieuse mixtes ${ }^{22}$. Si certaines organisations de femmes se définissent avant tout comme philanthropiques ou engagées dans l'action sociale, d'autres luttent explicitement pour l'émancipation. La présence de femmes juives vaut d'ailleurs à ces mouvements le soupçon d'être " enjuivés ». Dans le sillage du mouvement des femmes, des femmes juives conquièrent de nouvelles positions dans la sphère publique, alors même qu'elles en ont été exclues au cours du XIXe siècle. Elles viennent directement se confronter aux hommes sur les mêmes terrains. Dans les années 1860, en plein débat sur l'éducation et la formation professionnelle des jeunes femmes, le nouvel accès à l'éducation bénéficie en particulier aux femmes

17. Ute Frevert, Frauen-Geschichte. Zwischen bürgerlicher Verbesserung und neuer Weiblichkeit, Frankfurt a. M., Suhrkamp, 1986.

18. Susannah Heschel, "Jüdisch-feministische Theologie und Antijudaismus in christlichfeministischer Theologie », in Leonore Siegele-Wenschkewitz (dir.), Feministische Theologie und die Verantwortung für die Geschichte, München, Christian Kaiser, 1988, p. 54-103.

19. Erving Goffman, L'arrangement des sexes [1977], trad. Hervé Maury, Paris, La Dispute, 2002.

20. Voir Benjamin Maria Baader, "Jewish Difference and the Feminine Spirit of Judaism in MidNineteenth-Century Germany », in Benjamin Maria Baader, Sharon Gillerman, Paul Lerner (dir.), Jewish Masculinities. German Jews, Gender, and History, Bloomington, Indiana UP, 2012, p. 50-71.

21. Il s'agit du mouvement de la « première vague », pour lequel l'obtention du droit de vote en 1918 fut un jalon déterminant. Voir Patrick Farges, Anne-Marie Saint-Gille (dir.), Le premier féminisme allemand 1848-1933. Un mouvement social de dimension internationale, Villeneuve-d'Ascq, PU du Septentrion, 2013. Voir également la contribution d'Anne-Laure Briatte dans le présent dossier.

22. Les premières cérémonies de Bat Mitzvah (version féminine de la Bar Mitzvah) furent célébrées dès le début du XIXe siècle, puis se généralisèrent dans le mouvement libéral de réforme du judaïsme. 
juives des classes moyennes, ce qui inclut les milieux orthodoxes : on constate une surreprésentation des jeunes femmes juives bénéficiant d'une éducation, par rapport aux femmes chrétiennes et par rapport aux jeunes hommes juifs. En 1897 par exemple, près de $60 \%$ des jeunes filles juives à Berlin fréquentent une Höhere Töchterschule (contre $9 \%$ des non juives). On relève des chiffres similaires pour Prague, Vienne ou Karlsruhe. À Vienne, les jeunes hommes juifs représentent $30 \%$ des lycéens, alors qu'environ $50 \%$ des lycéennes sont juives.

L'émancipation a lieu aussi sur le terrain des études supérieures, auxquelles les femmes accèdent en 1897 en Autriche et en 1908 en Prusse ${ }^{23}$ : les cursus de médecine, de droit et de sciences humaines deviennent autant de voies d'accès à l'émancipation. On peut citer : Marie Munk (1885-1978), première femme docteure en droit en 1911, puis première avocate à Berlin en 1924; Selma Stern (18901981), fille de médecin, qui obtient en 1913 son doctorat d'histoire à Munich; ou Rahel Hirsch (1870-1953), petite-fille du rabbin Samson Raphael Hirsch, qui, docteure en médecine, est la troisième femme en Allemagne à être nommée sur une chaire professorale en 1913. L'ouverture des études supérieures connaît plusieurs étapes, qui correspondent à autant de cercles de protection autour du noyau de pouvoir (masculin) : d'abord auditrices libres dès les années 1860, les femmes sont autorisées à s'inscrire à l'Université, puis à y obtenir des diplômes. L'accès des femmes - juives de surcroît - à l'Université suscite d'importants mécanismes antiféministes et antisémites de défense, parce qu'il remet en cause les fondements mêmes du fonctionnement universitaire : cooptation et reproduction.

Ces nouvelles situations brisent certaines pratiques de l'entre-soi. La lutte de territoires est particulièrement âpre chez les médecins, les ingénieurs et les juristes. Concernant les autres secteurs du marché du travail, il est difficile d'avoir une vision claire de l'emploi des femmes juives, notamment parce que les codes bourgeois invisibilisent le travail des femmes. Dans les zones rurales, les femmes restent impliquées dans des formes d'économies familiales. En 1882 en Prusse, $11 \%$ des femmes juives sont enregistrées comme ayant un emploi (contre une moyenne de $21 \%$ pour l'ensemble des femmes). En 1907, après introduction de la catégorie statistique de mithelfende Familienangehörige (membre aidant de la famille), ces chiffres atteignent respectivement 18 et $31 \%$ (soit la moitié des femmes en âge de travailler). Enfin, on constate, dans la grande et la petite bourgeoisie, un phénomène de célibat féminin - et donc d'autonomie en particulier à Berlin ${ }^{24}$.

23. Voir Elisabeth Dickmann, Eva Schöck-Quinteros (dir.), Barrieren und Karrieren. Die Anfänge des Frauenstudiums in Deutschland, Berlin, Trafo, 2000; Patricia Mazón, Gender and the Modern Research University: The Admission of Women to German Higher Education, 1865-1914, Stanford, Stanford UP, 2003 ; Alison Rose, Jewish Women in Fin de Siècle Vienna, Austin, University of Texas Press, 2008. Il y a des différences régionales: I'accès aux études supérieures a lieu en 1900 dans le Bade, en 1903 en Bavière, en 1904 dans le Wurtemberg, en 1906 en Saxe, en 1907 en Saxe-WeimarEisenach (actuelle Thuringe), en 1908 en Prusse, en Hesse et en Alsace-Lorraine, et finalement en 1909 dans le Mecklembourg-Schwerin.

24. Schüler-Springorum, Geschlecht und Differenz, p. 53-56. 


\section{Genre et Bürgerlichkeit}

Ainsi l'histoire des femmes juives s'est-elle concentrée sur le statut et la position sociale des femmes. Elle les a réintégrées dans le récit historique, sans toutefois interroger les aspects relationnels du genre. Or l'égalité en droit acquise dans le dernier tiers du XIX siècle modifie en profondeur la vie quotidienne ainsi que les structures familiales des Juifs et des Juives dans le monde germanophone. Le genre fait partie des grandes questions traversant le XIXe siècle allemand, au même titre que la "question sociale ». Selon I'historienne Claudia Bruns, à mesure que les différences sociales propres à une société d'ordres s'amenuisent avec l'avènement de la société industrielle, la différenciation genrée gagne en importance, jusqu'à devenir le principe dominant de différenciation sociale. Les revendications féministes touchent ainsi au cœur même de la hiérarchie sociale ${ }^{25}$ et les questions centrales de l'histoire juive, comme la modernisation, la religiosité, l'embourgeoisement (Verbürgerlichung), l'ascension sociale ou l'exclusion, gagnent à être relues sous l'angle du genre.

Au premier rang des grandes dynamiques sociales propres à I'histoire des Juifs et Juives, il y a un processus $d^{\prime}$ '« entrée en bourgeoisie " sans précédent ${ }^{26}$ : dès 1871, environ deux tiers des Juifs et Juives d'Allemagne font partie de la classe moyenne bourgeoise, en particulier de la petite bourgeoisie commerçante. Cette évolution se traduit par une délocalisation des espaces de vie vers les quartiers bourgeois des grandes villes, par un accès facilité à certaines sphères professionnelles et par une modification des pratiques religieuses. En 1871, $80 \%$ des Juifs et Juives d'Allemagne résident à la campagne ou bien dans des villes petites ou moyennes. Quarante ans plus tard, plus de $50 \%$ sont citadins ( $25 \%$ pour la seule ville de Berlin). Le phénomène des conversions, apparu dès le début du XIXe siècle, en particulier dans les grandes villes, est un phénomène conjoint des élites et des classes juives paupérisées. Le processus d'embourgeoisement par le biais d'une apparition de modes de vie bourgeois (que Manfred Hettling nomme « Bürgerlichkeit ${ }^{27}$ ») est éminemment genré et la bourgeoisie allemande de la seconde moitié du XIX ${ }^{e}$ siècle se constitue en espace social ouvert, déterminé par des pratiques culturelles qui expriment à la fois des valeurs, une vision de la famille, des cultures alimentaires et de consommation, et des relations de genre.

25. Claudia Bruns, Politik des Eros. Der Männerbund in Wissenschaft, Politik und Jugendkultur (18801934), Köln/ Weimar/ Wien, Böhlau, 2008, p. 49.

26. Le terme est emprunté à Daniel Azuélos : L'entrée en bourgeoisie des Juifs allemands ou le paradigme libéral (1800-1933), Paris, PU Paris-Sorbonne, 2003. Voir aussi Shulamit Volkov, «Die Verbürgerlichung der Juden in Deutschland als Paradigma », in id. (dir.), Jüdisches Leben und Antisemitismus im 18. und 19. Jahrhundert, München, Beck, 1990, p. 111-130; Simone Lässig, Jüdische Wege ins Bürgertum: Kulturelles Kapital und sozialer Aufstieg im 19. Jahrhundert, Göttingen, Vandenhoeck \& Ruprecht, 2004; Benjamin Maria Baader, Gender, Judaism, and Bourgeois Culture in Germany, 1800-1870, Bloomington, Indiana UP, 2006.

27. Manfred Hettling, «"Verbürgerlichung" und "Bürgerlichkeit". Möglichkeiten und Grenzen für die deutschen Juden im 19. Jahrhundert », in Angelika Schaser, Stefanie Schüler-Springorum (dir.), Liberalismus und Emanzipation. In- und Exklusionsprozesse im Kaiserreich und in der Weimarer Republik, Stuttgart, Franz Steiner, 2010, p. 196-197. 
La catégorie du genre apporte un éclairage nouveau à la dialectique assimilation/dissimilation. Alors que sur le plan religieux, les différences de genre s'amenuisent au cours du siècle, les rôles de genre dans la sphère quotidienne, au contraire, se renforcent. Avec l'entrée en modernité, si certaines structures familiales traditionnelles sont rompues et si la vie publique et privée, des femmes comme des hommes, s'en trouve radicalement modifiée, les femmes juives restent chargées de la vie domestique, notamment de la tenue d'une cuisine casher (embourgeoisée) ou de la célébration (sécularisée) des fêtes juives. Ce sont les femmes qui façonnent la classe moyenne juive germanophone : d'une part elles dictent les codes de la vie sociale; d'autre part elles préservent une vie de famille distinctement juive ${ }^{28}$. On associe souvent le « judaïsme culinaire » à une forme de désaffiliation religieuse et d'assimilation; or les cultures culinaires et alimentaires - comme d'autres pratiques quotidiennes - ont justement permis de préserver et transmettre un sentiment d'appartenance au judaïsme, tout en le modifiant et en le « domestiquant ${ }^{29}$ ».

Le genre permet aussi de réévaluer les espaces d'actions respectifs des femmes et des hommes, ainsi que la distinction public-masculin vs privé-féminin. Disjoints à certains égards, le domestique et le public sont en réalité fortement imbriqués. La société civile bourgeoise en construction met en avant une masculinité indépendante et autonome, libérée de certaines entraves sociales afin de poursuivre des intérêts économiques. Dans le même temps, la féminité se constitue comme reposant sur les qualités domestiques de mère ou d'épouse ${ }^{30}$. Si I'historiographie a retenu le fait que les femmes juives s'assimilèrent plus lentement que les hommes, on peut se demander si, ce faisant, elles n'ont pas contribué à une meilleure intégration sociale de ces derniers : en cultivant une judéité domestique positive, les femmes ont sans doute permis l'exploration par les hommes de nouveaux possibles sociaux.

Par-delà les réussites socio-économiques, les Juifs et Juives restent toutefois des outsiders et le processus d'émancipation fut d'emblée ambivalent. Débutant à la fin du XVIII siècle et conduisant à une égalité juridique, l'émancipation en droit a fait disparaître les différences extérieures visibles entre Juifs et non-Juifs. Dans les représentations et discours institutionnels, les Juifs continuent cependant à ne pas être considérés comme des semblables. Au contraire même, ce n'est qu'après l'obtention de l'égalité en droit que l'antisémitisme s'impose en « code culturel $^{31} »$. La nouvelle position sociale des Juifs et Juives s'accompagne ainsi

28. Marion A. Kaplan (dir.), Geschichte des jüdischen Alltags in Deutschland. Vom 17. Jahrhundert bis 1945. München, Beck, 2003.

29. Monika Richarz, « Der jüdische Weihnachtsbaum - Familie und Säkularisierung im deutschen Judentum des 19. Jahrhunderts », in Michael Grüttner, Rüdiger Hachtmann, Heinz-Gerhard Haupt (dir.), Geschichte und Emanzipation, Frankfurt a. M., Campus, 1999, p. 275-289.

30. Paula E. Hyman, Gender and Assimilation in Modern Jewish History: the Roles and Representation of Women, Seattle, University of Washington Press, 1995; Isabel V. Hull, Sexuality, State, and Civil Society in Germany, 1700-1815, Ithaca, Cornell UP, 1996.

31. Shulamit Volkov, Antisemitismus als kultureller Code - Zehn Essays, München, Beck, 2000. 
de la construction de réseaux de sociabilité juive dans des espaces séparés ${ }^{32}$, mais aussi et surtout, d'opérations de marginalisation recouvrant des enjeux raciaux et genrés : faiblesse supposée des hommes juifs, soupçon de réversion de l'ordre genré, d'homosexualité, et, par conséquent, de mise en danger du "bon" fonctionnement de la nation et de ses institutions, dont la famille. C'est bien l'affirmation d'un ordre sexuel et genré national qui rassemble différents discours antisémites, comme le souligne Jennifer Meyer :

\footnotetext{
L'apparente contradiction entre la désexualisation, la pathologisation sexuelle et I'hypersexualisation des juives et juifs révèle en réalité le caractère polymorphe et totalisant de l'antisémitisme à l'œuvre dans les débats véhéments sur l'ordre des sexes ${ }^{33}$.
}

Une histoire des rapports sociaux de genre doit permettre d'éclairer la variabilité tant des constructions du féminin que de celles du masculin. À y regarder de plus près, le masculin et la masculinité, longtemps considérés comme allant de soi, sont en réalité variables dans le temps et l'espace social.

\section{Histoire du genre et masculinités germano-juives}

L'historiographie sur les masculinités germano-juives est relativement récente. Elle est «fille de l'histoire du genre et petite-fille de l'histoire des femmes », selon Deborah Hertz ${ }^{34}$. L'un des premiers travaux en histoire des masculinités est l'œuvre de I'historien américain d'origine juive allemande George Mosse, qui s'intéressa au milieu des années 1990 aux conditions d'émergence de critères normatifs du masculin dans l'espace germanophone au cours du grand XIX siècle $^{35}$. II manquait toutefois à ses intuitions un fondement dans les sources : le colloque de San Diego en 2005, « Jewish Masculinities in Germany ${ }^{36}$ », est venu pallier ce manque.

L'histoire des masculinités est relationnelle, multidimensionnelle et intersectionnelle, et elle intègre différentes formes de domination : domination des hommes sur les femmes, domination de certains hommes sur d'autres hommes, mais aussi rapports de domination sociale et raciale ${ }^{37}$. La masculinité correspond, à une époque et dans un contexte social donnés, à un ensemble d'éléments reconnus comme devant être le propre des hommes, qui subissent une

32. Till van Rahden (dir.), Juden, Bürger, Deutsche, Tübingen, Mohr, 2001.

33. Jennifer Meyer, «La racialisation de l'ordre des sexes dans l'Allemagne de l'entre-deux-guerres. Trois cas d'antisémitisme genré », in GenERe (dir.), Épistémologies du genre. Croisement des disciplines, intersections des rapports de domination, Lyon, ENS éditions, 2018, p. 216.

34. Deborah Hertz, "Männlichkeit und Melancholie im Berlin der Biedermeierzeit », in Heinsohn, Schüler-Springorum, Jüdische Geschichte als Geschlechtergeschichte, p. 291.

35. George L. Mosse, The Image of Man. The Creation of Modern Masculinity, Oxford/ New York, Oxford UP, 1996 (L'Image de I'homme : l'invention de la virilité moderne, trad. Michèle Hechter, Paris, Abbeville, 1997).

36. Voir Baader, Gillerman, Lerner, Jewish Masculinities.

37. Thomas Kühne (dir.), Männergeschichte - Geschlechtergeschichte. Männlichkeit im Wandel der Moderne, Frankfurt a. M., Campus, 1996; Jürgen Martschukat \& Olaf Stieglitz, Geschichte der Männlichkeiten, Frankfurt a. M., Campus, 2008. 
injonction à les acquérir, les entretenir et les défendre ${ }^{38}$. L'histoire des masculinités s'appuie sur deux hypothèses : la dimension performative du genre ${ }^{39}$ et la notion de " masculinité hégémonique », empruntée à la sociologue Raewyn Connell, qui permet de penser une hiérarchisation des masculinités ${ }^{40}$. Le modèle hégémonique sert, dans un contexte donné, de référence, et les formes de masculinité dominées, alternatives, marginales ou complices se positionnent par rapport à lui. La «masculinité hégémonique » est donc un ensemble de pratiques, rites et normes mis en place par ceux qui bénéficient des avantages conférés par I'hégémonie. Ce type de configuration se constitue en tension non seulement avec la féminité, mais également avec d'autres formes de masculinité, plus ou moins complices, mais marginalisées en raison de rapports de classe, de race ou de sexualité. En Allemagne, la masculinité hégémonique s'incarne à partir des guerres napoléoniennes (1806-1815) dans un idéal de virilité guerrière reposant sur la capacité à défendre son honneur, sa famille et la nation (Wehrhaftigkeit). Pendant un temps, ce modèle entre en conflit avec d'autres idéaux masculins contemporains, comme la contention, la bienveillance ou la sensibilité masculines (sanfte Männlichkeit ${ }^{41}$ ). Au début de la Première Guerre mondiale toutefois, la militarisation de l'idéal masculin est devenue hégémonique.

La grande diversité des arrangements genrés du masculin participe de la façon dont les Juifs et Juives d'Allemagne ont négocié les bouleversements politiques et sociaux entre 1867 et 1933. La conquête par les femmes de nouveaux territoires a suscité, nous l'avons vu, diverses réactions de défense. Ce même raisonnement vaut pour les hommes juifs qui, dans un contexte d'embourgeoisement, accèdent à de nouveaux espaces socio-professionnels : cette lutte de territoires affecte leur identité masculine. Deux caractéristiques sont régulièrement rejetées hors du champ de la masculinité hégémonique : l'efféminement ${ }^{42}$ et l'« enjuivement ». Des années 1880 aux débuts de la République de Weimar, apparaissent des stratégies discursives d'exclusion de l'"autre", en particulier s'il est juif. Avec l'avènement d'un antisémitisme racial dans l'Allemagne wilhelminienne et l'Autriche-Hongrie de la fin-de-siècle, le corps des hommes juifs, et donc la masculinité juive, deviennent des enjeux nationaux. À travers un système complexe de justifications pseudo-

38. Pour la recherche française, voir Anne-Marie Sohn, "Sois un homme! » La construction de la masculinité au XIX siècle, Paris, Seuil, 2009; id. (dir.), Une Histoire sans les hommes est-elle possible?, Lyon, ENS éditions, 2013.

39. C'est-à-dire que l'identité de genre comme construction sociale se cristallise par la répétition de mises en scène genrées de soi dans un but de reconnaissance sociale. Voir Candace West, Don $\mathrm{H}$. Zimmerman, « Doing Gender », Gender \& Society 1, 1987, p. 125-151.

40. Raewyn Connell, Masculinités. Enjeux sociaux de l'hégémonie, éd. M. Hagège \& A. Vuattoux, Paris, Éditions Amsterdam, 2014.

41. Karen Hagemann, 'Mannlicher Muth und Teutsche Ehre': Nation, Militär und Geschlecht in Preußen zur Zeit der Antinapoleonischen Kriege, Paderborn, Schöningh, 2002; Anne-Charlott Trepp, Sanfte Männlichkeit und selbständige Weiblichkeit: Frauen und Männer im Hamburger Bürgertum 1770-1840, Göttingen, Vandenhœck \& Ruprecht, 1996.

42. Au tournant du siècle, on observe une fascination morbide pour la décadence des valeurs et une peur du déclin par la « féminisation ». Voir Annette Runte, Eva Werth (dir.), Feminisierung der Kultur? Krisen der Männlichkeit und weibliche Avantgarden, Würzburg, Königshausen \& Neumann, 2007. 
scientifiques, le corps juif est constitué comme"autre" par une opération récurrente de comparaison plus ou moins explicite avec le corps national. Sander Gilman, pour l'Allemagne, et Klaus Hödl, pour l'Autriche, ont montré que l'efféminement et la faiblesse supposée du corps juif masculin étaient le produit de stratégies reposant sur la construction de dichotomies (allemand/ juif, chrétien/ juif, masculin/ féminin) et sur un système d'analogies (féminin-juif, malade-juif ${ }^{43}$ ). Le corps juif masculin est scruté : il s'agit d'y déceler les stigmates de la maladie, une gestuelle ou encore une mollesse prétendument féminines ${ }^{44}$.

S'éloignant de l'étude historique des discours plus ou moins ouvertement antisémites, de nouvelles approches ont analysé des sources juives et une vision " de l'intérieur ». Ainsi des conceptions alternatives de l'identité masculine ont-elles été mises au jour au sein de la tradition juive, notamment par les travaux de Daniel Boyarin sur l'anti-héroïsme, que l'auteur nomme «Edelkayt ${ }^{45}$ ». II estime que la culture juive est-européenne a produit une " masculinité sensible », spécifiquement juive. Si l'on suit Boyarin, on peut considérer la forme de masculinité issue du judaïsme rabbinique classique - fondée sur les valeurs de modestie, de piété, de sens de la famille ou de refus de la violence - comme un contre-modèle à une conception " occidentale ", nationaliste et martiale de la masculinité.

Si l'on se place du côté de l'histoire sociale à présent, il est intéressant de constater que ces représentations ont produit des pratiques sociales de masculinité " complice », notamment chez les jeunes hommes juifs, p. ex. chez les étudiants ${ }^{46}$. Comme d'autres milieux bourgeois, le milieu étudiant du Reich wilhelminien puis de la République de Weimar est perméable aux discours et pratiques antisémites ${ }^{47}$. Des corporations juives, nées à la fin du XIX siècle de l'exclusion des étudiants juifs des corporations traditionnelles, tentent de remédier à la position d'outsiders des jeunes Juifs ${ }^{48}$. En 1896 est fondé le Kartell-Convent

43. Sander L. Gilman, The Jew's Body, New York, Routledge, 1991 ; Klaus Hödl, Die Pathologisierung des jüdischen Körpers. Antisemitismus, Geschlecht und Medizin im Fin de Siècle, Wien, Picus, 1997.

44. C'est dans ce contexte que s'inscrit la publication en 1893 de la nouvelle d'Oskar Panizza, Der operierte Jud, exemplaire d'une série de productions littéraires posant l'altérité raciale de l'homme juif. Voir Arnon Hampe, "L'assimilation comme travestissement : masculinité hégémonique et antisémitisme dans Der Operierte Jud ("Le Juif opéré") d'Oskar Panizza », in Maxime Cervulle, Patrick Farges, Anne Isabelle François (dir.), Marges du masculin : exotisation, déplacements, recentrements, Paris, L'Harmattan, 2015, p. 111-127.

45. Daniel Boyarin, Unheroic Conduct: The Rise of Heterosexuality and the Invention of the Jewish Man, Berkeley, University of California Press, 1997.

46. Les comportements rituels étudiants avaient été observés par Norbert Elias : Studien über die Deutschen. Machtkämpfe und Habitusentwicklung im 19. und 20. Jahrhundert, Frankfurt a. M., Suhrkamp, 1989. Voir aussi Lisa F. Zwicker, Duelling Students: Conflicts, Masculinity, and Politics in German Universities, 1890-1914, Ann Arbor, University of Michigan Press, 2011.

47. Norbert Kampe, Studenten und „Judenfrage“ im Deutschen Kaiserreich: die Entstehung einer akademischen Trägerschicht des Antisemitismus, Göttingen, Vandenhœck \& Ruprecht, 1988.

48. Miriam Rürup, « Auf Kneipe und Fechtboden. Inszenierung von Männlichkeit in jüdischen Studentenverbindungen in Kaiserreich und Weimarer Republik », in Martin Dinges (dir.), Männer-Macht-Körper. Hegemoniale Männlichkeiten vom Mittelalter bis heute, Frankfurt a. M., 
der Verbindungen deutscher Studenten jüdischen Glaubens, né de la fusion de cinq corporations juives. Dans la mouvance sioniste, une autre fusion de corporations donne naissance en 1901 au Bund Jüdischer Corporationen, qui lui-même s'unit au Kartell Zionistischer Verbindungen, pour former, à la veille de la guerre, le Kartell Jüdischer Verbindungen. Ces corporations sont le lieu d'un "devenir-homme" et d'un "devenir-allemand". Les sorties à la taverne (Kommers), la pratique de l'escrime (Mensur), les rites initiatiques, l'identification aux couleurs et aux mots d'ordre (Komment) rythment le quotidien de ces corporations. Certaines pratiques visent à façonner la gestuelle, les expressions faciales ainsi que le langage et les intonations : c'est le corps tout entier qui est discipliné. Dans le même temps, il s'agit d'une pratique distinctement juive, entre soi. On est ici confronté à une logique de riposte, « complice » de l'hégémonie (au sens connellien). Au sein des lieux sociaux structurés par la compétition et l'émulation, où l'identité se forge dans l'interaction avec les pairs, par le truchement de rites d'initiation, d'un esprit de corps, de jeux de pouvoir et de tensions, des formes de masculinité sont constamment produites et transmises. Loin d'être anodines, les frictions font partie d'un système de compétition au sein d'univers exclusivement masculins. Le corps, vecteur et réceptacle de ces frictions, y joue un rôle central pour asseoir une position dans la hiérarchie genrée, et donc sociale. L'éducation du corps aux normes de genre est un ressort puissant de socialisation : la virilité comme idéal se démontre et se défend; en retour, la masculinité risque à tout moment d'être remise en cause.

La question de la masculinité des hommes juifs se pose régulièrement dans le contexte militaire ${ }^{49}$. Pour un jeune homme juif, il est d'autant plus important de prouver sa virilité que la stigmatisation antisémite insiste sur sa faiblesse supposée. Dans les milieux sionistes, on tente ainsi de contrer les stéréotypes : le « Nouvel Homme Juif » répond point par point au stigmate. Afin de rompre avec l'image du Juif chétif et intellectualisé, le « Nouveau Juif » est robuste et fort, stratège et prêt à se battre, courageux et viril. Contre le stigmate d'efféminement et d'homosexualité, il incarne I'hétérosexualité. Proche de Theodor Herzl, le médecin aliéniste Max Nordau ${ }^{50}$, influencé par le courant hygiéniste, développe la thèse de la régénération juive lors du deuxième Congrès sioniste à Bâle en 1898. Celle-ci comprend évidemment l'exercice corporel, mais également les travaux physiques, en particulier agricoles. II définit ainsi un « judaïsme du

Campus, 2005, p. 141-156; id., Ehrensache. Jüdische Studentenverbindungen an deutschen Universitäten 1886-1937, Göttingen, Wallstein, 2008.

49. Gregory A. Caplan, « Germanising the Jewish Male. Military Masculinity as the Last Stage of Acculturation », in Rainer Liedtke, David Rechter (dir.), Towards Normality? Acculturation and Modern German Jewry, Tübingen, Mohr Siebeck, 2003; Derek Penslar, Jews and the Military: A History, Princeton (NJ), Princeton UP, 2013. Sur le lien entre genre et Nation, voir Patrick Farges, Brice Chamouleau, « Nation », in Juliette Rennes (dir.), Encyclopédie critique du genre, Paris, La Découverte, 2016, p. 400-408.

50. Né en Hongrie, Max Nordau (1849-1923) se réinvente à son arrivée à Berlin en 1873. Il change son nom de Simon Südfeld en Max Nordau. Voir Delphine Bechtel, Dominique Bourel, Jacques Le Rider (dir.), Max Nordau (1849-1923). Critique de la dégénérescence, médiateur franco-allemand, père fondateur du sionisme, Paris, Cerf, 1996. 
muscle » (Muskeljudentum), évoquant les torses saillants, les corps d'athlètes et la hardiesse des regards, et réclamant une jeunesse agile, souple et musclée, à l'image des ancêtres mythiques : les Hasmonéens, les Maccabées ou Shimon Bar Kokhba ${ }^{51}$. Cette représentation du « Muskeljude » est par la suite largement diffusée dans la culture populaire (almanachs, cartes postales).

La Première Guerre mondiale donne aux Juifs l'occasion de prouver leur germanité et de s'affranchir des obstacles à la pleine égalité ${ }^{52}$. Ils sont déterminés à faire la preuve de leur camaraderie et de leur patriotisme. Cette volonté transcende largement les différences entre orthodoxes, sionistes, assimilés ou convertis. Or en octobre 1916, le " comptage des Juifs », dont les résultats ne sont de surcroît pas rendus publics, renforce les stéréotypes antisémites, malgré l'évidence de la participation des hommes juifs à l'effort de guerre ${ }^{53}$. Les accusations selon lesquelles les Juifs auraient été des " planqués » (Drückeberger ${ }^{54}$ ) conduisent à la création en 1919 du Reichsbund jüdischer Frontsoldaten, dont l'objectif est de défendre l'honneur des vétérans juifs. À la fin des années 1920, l'organisation compte près de la moitié des quelque 8000 vétérans juifs d'Allemagne ${ }^{55}$. Elle cultive un style commémoratif héroïque et nationaliste, et exhorte ses membres à affronter directement l'extrême-droite dans une logique d'Abwehr. Cette stratégie a une influence considérable sur le comportement des ex-soldats juifs dans l'entre-deux-guerres ${ }^{56}$. Mais le statut de « poilu » ne protège plus après 1933, nombre de vétérans ne verront pas le danger.

\section{Conclusion : des « régimes de genre »}

Au même titre que la micro-histoire, l'histoire du quotidien ou l'anthropologie historique, l'histoire du genre conduit l'historien-ne à considérer la société à hauteur d'être humain, comme une combinatoire d'affiliations et d'interactions sociales. Les constellations quotidiennes de l'expérience individuelle fournissent la matière à une histoire du genre intégrative qui tienne compte de la variété des expériences vécues, différentes selon le sexe, l'âge, la classe sociale ou la localisation géographique. En ce sens, I'histoire du genre participe pleinement

51. Max Nordau, Zionistische Schriften, Cologne/ Leipzig, Jüdischer Verlag, 1909, p. 379-388.

52. Tim Grady, The German-Jewish Soldiers of the First World War in History and Memory, Liverpool, Liverpool UP, 2011 ; id., A Deadly Legacy: German Jews and the Great War, New Haven (CT), Yale UP, 2017.

53. Jacob Rosenthal, 'Die Ehre des jüdischen Soldaten'. Die Judenzählung im Ersten Weltkrieg und ihre Folgen, Frankfurt a. M., Campus, 2007.

54. Voir Volker Ulrich, "Fünfzehntes Bild: Drückeberger », in Julius Schoeps, Joachim Schlör (dir.), Bilder der Judenfeindschaft. Antisemitismus, Vorurteile und Mythen, Augsburg, Weltbild, 1999, p. 210-217.

55. Ulrich Dunker, Der Reichsbund jüdischer Frontsoldaten, 1919-1938. Geschichte eines jüdischen Abwehrvereins, Düsseldorf, Droste, 1977.

56. Voir Michael J. Geheran, "Remasculinizing the Shirker: The Jewish Frontkämpfer under Hitler », Central European History 51, 2018, p. 440-465. 
de l'écriture d'une histoire des sociétés ${ }^{57}$. Le genre est donc bien une " catégorie utile » de l'histoire juive allemande, à condition de travailler le concept et de le confronter aux sources. Revenant sur ses réflexions séminales, Joan Scott écrivait en 2009:

Que le genre reste ou non une catégorie utile d'analyse - historique ou autre - me paraît reposer moins sur le terme lui-même que sur les utilisations critiques que nous continuons d'en faire. [...] Je pense que le genre ne peut demeurer utile que [...] s'il est pris comme une invitation à réfléchir sur un mode critique à la manière dont les significations des corps sexués sont produites en relation les unes avec les autres, à s'interroger sur la manière dont ces significations se déploient et se modifient ${ }^{58}$.

L'histoire du genre est productive si elle est relationnelle (combinant une histoire des femmes et des hommes), et intersectionnelle, c'est-à-dire si elle reconnaît la « consubstantialité » des rapports de pouvoir (classe, âge, place dans la parenté, race $^{59}$ ). Ses préoccupations doivent inclure une réflexion sur les appartenances et affiliations multiples imbriquées.

Le genre est une catégorie « récalcitrante » de l'analyse historique : il imbrique des aspects ambigus de libéralisation/répression, de consentement/domination, de privé /public. Pour désigner ces configurations, le médiéviste Didier Lett propose la notion de "régime de genre », qui permet de penser la coexistence dans le temps de plusieurs agencements de genre parfois concurrents entre eux :

Un régime de genre peut être défini comme un agencement particulier et unique des rapports de sexe dans un contexte historique, documentaire et relationnel spécifique. Plusieurs régimes peuvent coexister dans une même période historique. Dépendant d'une série d'opérations de contextualisation, ils sont instables, sujets à variation lorsque l'historien change de documentation, déplace son regard vers d'autres acteurs ou dès que les relations entre les acteurs observés se modifient ${ }^{60}$.

Lett insiste particulièrement sur la situation documentaire de l'historien-ne qui, par son choix de sources, façonne ses lunettes genrées. C'est en ce sens que le genre aide à rendre compte de la complexité des vies juives dans l'Allemagne des années 1867-1933.

57. Voir Karin Hausen, Geschlechtergeschichte als Gesellschaftsgeschichte, Göttingen, Vandenhœck \& Ruprecht, 2012

58. Joan W. Scott, « Le genre : une catégorie d'analyse toujours utile? », Diogène 225 (1), 2009, p. 10.

59. Elsa Galerand, Danièle Kergoat, «Consubstantialité vs intersectionnalité? À propos de l'imbrication des rapports sociaux », Nouvelles pratiques sociales 26 (2), 2014, p. 44-61.

60. Didier Lett, "Les régimes de genre dans les sociétés occidentales de l'Antiquité au XVIle siècle », Annales. Histoire, Sciences Sociales 67 (3), 2012, p. 565-566. 
